CMHP. Reducing child poverty in both lone- and two-parent households also decreased prevalence in CMHP (7.9\% [7.0, $8.9]$ ), but resulted in smaller reductions in inequality due to family structure (RR 1.63; RD 4.60). Sensitivity analyses showed that associations between exposure, mediator and outcome were comparable across MCS sweeps.

Conclusion Inequalities in CMHP between lone- and twoparent families in the UK are large, but could be reduced by tackling income inequality.

\section{OP85 PREVALENCE OF PTSD AND COMORBIDITY WITH ANXIETY AND DEPRESSION IN A POPULATION-BASED SURVEY OF WOMEN FOLLOWING CHILDBIRTH}

${ }^{1} S$ Harrison*, ${ }^{2} S$ Ayers, ${ }^{1} \mathrm{MA}$ Quigley, ${ }^{1} \mathrm{~F}$ Alderdice. 'National Perinatal Epidemiology Unit, University of Oxford, Oxford, UK; ${ }^{2}$ City, University of London, London, UK

\subsection{6/jech-2019-SSMabstracts.88}

Background Post-traumatic stress disorder (PTSD) can occur after the experience of real or perceived trauma during childbirth or the postpartum period. Estimates of the prevalence and correlates of PTSD following childbirth vary widely in the literature. The aim of this study was to explore: prevalence of PTSD; comorbidity between PTSD, anxiety and depression; and association between PTSD and sociodemographic characteristics in a large sample of women six months after childbirth.

Methods The study was a cross-sectional population-based questionnaire survey of 16,000 women selected at random from birth registrations. The women had given birth in England during October 2017 and were six months postpartum at the time of the survey. The women received a postal questionnaire, which they could complete on paper, by telephone or online. The questionnaire included validated measures of PTSD (Primary Care PTSD Screen (PC-PTSD)), anxiety (Generalised Anxiety Disorder Scale-2) and depression (Edinburgh Postnatal Depression Scale) in addition to general questions covering health and care during pregnancy, labour and birth, and the postnatal period. Prevalence of PTSD, anxiety and depression was estimated with 95\% confidence intervals; association between PTSD and sociodemographic characteristics was explored using logistic regression. Survey weights based on characteristics associated with response were applied to the data.

Results Questionnaires were returned by 4,509 women. The mean age of the women was 32 years ( $\mathrm{sd}=5$ years) and the majority were married (64\%), born in the UK (77\%), and from White British backgrounds (76\%). One in ten women (9.4\%,95\%CI:8.6-10.3) scored above the cut-off on the PCPTSD, reporting at least three of the four symptoms of PTSD (re-experiencing, emotional numbing, avoidance, hyperarousal); $2.5 \%$ of women indicated that their symptoms were specifically related to their childbirth experience (95\% CI:2.1-3.0). The prevalence of anxiety was significantly higher in women with PTSD (score $>3$ ) (45.0,95\%CI:40.150.0) compared to women without PTSD (score $<3$ ) (9.9\%,95\%CI:9.0-10.9). Similarly, the prevalence of depression was significantly higher in women with PTSD (59.3\%,95\%CI:54.3-64.1) compared to women without PTSD (10.7\%,95\%CI:9.8-11.8). Regression analyses indicated that women who were younger, unmarried and born in the UK were more likely to report PTSD.
Conclusion The survey findings indicate a significant proportion of women were experiencing PTSD six months after childbirth. There was high comorbidity between PTSD, anxiety and depression with younger, unmarried, UK-born women being most at risk. Current guidelines recommend screening for anxiety and depression during the postnatal period; a brief screen for PTSD should be included to ensure women with symptoms are identified and offered focused interventions.

\section{OP86 PREVALENCE OF DEPRESSION AMONG PEOPLE WITH DIABETES; A COMPARATIVE ANALYSIS OF ADULTS ACROSS THREE HEALTH SYSTEMS USING NATIONALLY REPRESENTATIVE DATA}

${ }^{1} \mathrm{~N}$ McGrath*, 'S McHugh, ${ }^{2} \mathrm{E}$ Toomey, ${ }^{1}$ PM Kearney. 'School of Public Health, University College Cork, Cork, Ireland; '2School of Psychology, National University of Ireland, Galway, Galway, Ireland

\subsection{6/jech-2019-SSMabstracts.89}

Background Cross country comparison facilitates examination of health-system and country-level similarities and differences on disease burden. Diabetes is a leading global health issue with outcomes influenced by psychological comorbidity. Diabetes is consistently associated with depression in different populations but any association is influenced by variations in prevalence, study design and measure used. We compared depressive symptom prevalence in older adults with and without diabetes across three health systems using a validated measure to examine the causal association between diabetes and depression.

Methods We used data of adults aged 50 years and older, from three nationally representative ageing datasets; The Irish Longitudinal Study on Ageing (TILDA), the English Longitudinal Study on Ageing (ELSA) and the Health and Retirement Study (HRS). Data were collected during 2009-2011 (TILDA), and 2010 (ELSA, HRS). Variables; country, gender, age, education level, marital status and smoking status, were selected a priori using literature and a directed acyclic graph. Variables were self-report, with the exception of 'country', which was linked to the original dataset. The 20-item (cut-off-score $>16$; TILDA) and 8-item CESD (cut-off-score $>3$;ELSA and HRS) were used to categorise depression. Prevalence was presented as a percentage with corresponding 95\% confidence intervals and group-specific differences presented using Pearson's chisquare test for categorical data and Student's t-test for continuous data. Binomial logistic regression examined the odds of depression by diabetes status, adjusting for pre-selected variables. Analyses of country-specific factors related to depression is ongoing. Data were analysed using Stata v15.

Results Diabetes prevalence varied across the countries (Ireland; 8\%[(95\%CI:7.5-8.6), England; 11\%(95\%CI:10.6-12.0), USA; 22\%[(95\%CI:21.7-22.8); $<<0.001])$ and was consistently higher among males than females (Ireland; 10\%(95\%CI:8.9$10.8)$ vs. $6 \%(95 \% \mathrm{CI}: 5.3-6.7)$, England; 13\%(95\%CI:12.4 $14.5)$ vs. $10 \%(95 \% \mathrm{CI}: 8.7-10.3)$, USA; 24\%(95\%CI:22.9-24.7) vs. $21 \%[(95 \% \mathrm{CI}: 20.3-21.8) ; \mathrm{p}<0.001])$. Depression prevalence was significantly higher among people with diabetes in all countries (Ireland; 12\%(95\%CI:9.8-15.1) vs. 10\%[(95\% CI:9.0-10.4), $\mathrm{p}=0.035]$, England; 34\%(95\%CI:31.0-37.0) vs. 22\%[(95\%CI:20.8-22.6):p<0.001], USA; 20\%(95\%CI:19.0$21.4)$ vs. $14 \%[(95 \% \mathrm{CI}: 13.6-14.7) ; \mathrm{p}<0.001]$, in females (Ireland; $19 \%(95 \% \mathrm{CI}: 14.6-23.9) \quad$ vs. $12 \%[(95 \% \mathrm{CI}: 11.0-12.9)$; $\mathrm{p}=0.001]$, England; 39\%(95\%CI:34.2-43.4) vs. $25 \%[(95 \%$ 